\title{
A Laboratory Excitation Technique to Test Road Bike Vibration Transmission
}

Julien Lépine, (julien.lepine@usherbrooke.ca), MSc student

Yvan Champoux, PhD (Engineering), Professor

Jean-Marc Drouet, PhD (Engineering), Professor

Department of Mechanical Engineering, Université de Sherbrooke, Sherbrooke, Qc, Canada

\section{ABSTRACT}

This paper describes a technique designed to measure the in-situ acceleration signals that will be used to drive a road simulator in the study of road bike vibration transmission in a laboratory setting. To measure the signals, a bike mounted by a cyclist and towed by a motor vehicle is used. A road simulator using actuators driven by a digital signal is described. The impulse response of the bike used to measure road data is convoluted with the road acceleration in order to obtain the required actuator signal. The reproduction capacity of the simulator is evaluated by comparing the frequency content as well as the time statistical parameters of the acceleration signal measurement on road to the acceleration obtained on the simulator. On a granular road with a broadband excitation spectrum, the vertical excitation obtained with the simulator adequately mimics the measured road acceleration. This technique can be used to compare vibration transmission characteristics among different road bikes.

\section{Keywords:}

Road excitation, bicycle dynamics, road vibration measurement, vibration replication

\section{Introduction}

Next to being lightweight, one of the most oft-mentioned desirable characteristics of a road bike is its capacity to filter vibrations caused by an uneven road surface and transmitted to the cyclist's hands, buttocks and feet. Riding a bicycle for hours on a rough road can be quite uncomfortable and can even cause non-traumatic injuries such as handlebar palsy associated with a nerve disorder of the hand and arm [1]. All major bike manufacturers are looking for ways to reduce transmitted vibration and improve the quality and comfort of the ride. With the goal of designing bicycles with an improved vibration filtration capacity, the importance of being able to assess bicycle vibration transmissibility (from the road to the cyclist) cannot be overemphasized. Surprisingly, very little research on the particular issue of measuring the vibration transmissibility of a road bicycle has been done to date [2-4]. 
From a practical standpoint, on-road measurement of bicycle transmissibility is a difficult task because excitation input such as force, displacement and acceleration at the tire-road interface cannot be easily measured. However, in a laboratory using a simulator testing rig, these inputs can be measured and bicycle transmissibility can be determined. The main challenge when using a laboratory technique for vibration testing is to get proper excitation in order to mimic realistic road excitations. This is even more important for bike vibration transmission studies due to certain non-linear behaviors of the cyclist's buttocks and hand-arm system [5-7]. For bike vibration transmission testing, both Richard [8] and Hasting et al. [3] have used a treadmill with an object glued on the belt in order to simulate impact. Champoux et al. [2] presented several techniques and instrumented components to study the dynamic behavior of a road bike. Nordstrom [9] proposed a method to rank mountain bike comfort using in-situ acceleration transmissibility between the rear axle and the saddle. However, no previous studies have revealed a technique to test road bikes with the main objective of providing a realistic duplication of various road excitations in a laboratory setting.

Using a laboratory technique with the participation of a cyclist to study vibration transmission is also of capital importance in obtaining realistic transmissibility measurement data, and provides many advantages. First, it must be emphasized that the cyclist obviously has a prevailing influence [8] on bike dynamics, considering the weight of the bike and the fact that the cyclist adds a fair amount of damping to a road bike. Second, to cope with a wide variety of riding conditions and road events, the cyclist must constantly shift position and posture on the bike. This considerably modifies the dynamic response of the bike [10] and adds a level of difficulty to the comparative measurement study. Third, in a sheltered laboratory environment it is possible to test all year long. And even more importantly, a large number of channel counts can be used without the cyclist having to carry extra loads or route wires along the bike frame, which would modify the bike’s dynamic response.

The primary purpose of this research study is to develop a simple technique to obtain road data signal inputs. The second purpose is to evaluate the quality of on-road replication when using these data with a road bike road vibration simulator developed in a previous study [11]. The main objective of the excitation technique proposed in this article is to provide a repeatable displacement profile that represents a typical road excitation. This excitation can then be used in future studies to compare bike transmissibility based on repeatable and realistic displacement excitations. 


\section{Section 1: Road Data Measurement Technique}

This first section describes a technique to get road data signals that will be used as inputs on the road bike simulator described in Section 2. The in-situ road data will also be used in this paper to evaluate the replication capability of the laboratory excitation technique.

\section{Methods}

Several road characterization techniques have been developed for the automotive industry. These methods can be separated in two overall categories: direct measurement of the pavement profile with various types of instrumentation [12] [13] and the use of mechanical devices or systems with acceleration measurements to infer the pavement profile [13-17]. The technique presented in this paper belongs to the second category.

In the technique proposed in this paper, the vertical $(Z)$ time acceleration signal $a(t)$ is measured at the left rear wheel drop-out. As shown in Figure 1, the measurement is done while the cyclist is riding a bike towed by a car. Acceleration measurement is provided by a PCB triaxial accelerometer model 356B11. The bike's front fork is attached with an appropriate connection to the rear bumper of the car. A dummy front hub attached to the bumper fixture allows the fork to rotate freely along the front axle. An instrumented stem [8] measures the leaning vertical force amplitude applied by the cyclist, who can then monitor this force amplitude and keep it at a constant value of $140 \mathrm{~N}$. The cyclist does not pedal and the bike chain has been removed to ensure that it does not disturb the acceleration signal. The tire pressure is set at 8 Bar. During the tests, the car speed is kept constant. The test is repeated four times over the same $1.5 \mathrm{~km}$ portion of the road at 3 different speeds: 26,30 and $36 \mathrm{~km} / \mathrm{h}$.

All transducer electric cables are routed along the frame and connected to the power supply and LMS SCADAS The recorder data acquisition system model SCR01 is installed in the car. The sample rate is set at $44 \mathrm{kHz}$ and throughput time data is recorded. The data are analyzed with LMS Test.Lab software. For this study, the frequency range of interest is between 8-100 Hz, which corresponds to the frequency band for which the hand-arm system is more sensitive [5][17]. A frequency resolution of $1 \mathrm{~Hz}$ is selected and a Hanning time window with 67\% overlap is used for averaging purposes. The recording time for each test was approximately 3 minutes, which allowed an average Power Spectrum Density spectrum (PSD) calculation using more than 500 frequency spectrums. 
Acceleration measurements performed on the same road at the bumper fixture show that the acceleration level on the bumper is $15 \mathrm{~dB}$ above the acceleration level measured on the bike's rear dropout. This indicates that the rear wheel bicycle dropout measurements are not significantly influenced by car vibrations transmitted through the front fork.

Frequencies along with time domain analyses are used in this paper. Time data distribution of the vertical ac coupled acceleration signal $a(t)$ and average time data parameters including the standard deviation $\sigma$, the root mean square $a_{\text {rms, }}$, the skewness $s$ and the kurtosis $\kappa$ will be calculated according to the following equations, respectively, where $T$ is the averaging time:

$$
\begin{gathered}
a_{\mathrm{rms}}=\sigma=\sqrt{\frac{1}{T} \int_{0}^{T}[a(t)]^{2} d t} \\
s=\frac{1}{T} \int_{0}^{T}\left[\frac{a(t)}{\sigma}\right]^{3} d t \\
\kappa=\frac{1}{T} \int_{0}^{T}\left[\frac{a(t)}{\sigma}\right]^{4} d t
\end{gathered}
$$

Note that these parameters are central moments around the mean value. The mean value of the acceleration is equal to zero because there is no net vertical displacement of the bike. This explains why the mean variable does not appear in Eqs (1-3). The skewness describes the symmetry of the distribution. In a normal distribution, the sample skewness value is equal to zero. A distribution with a positive skewness has a right tail longer than the left tail. In this study, this would mean that there are a small number of high level positive acceleration events (upward) that create a positive skew in the distribution. The kurtosis is a descriptor of the shape of a probability distribution and is related to its "peakedness". A normal distribution will have a kurtosis value of 3. A kurtosis greater than 3 would mean the acceleration sample values are predominantly distributed around the mean, giving the distribution a greater peak. The time parameters are used here to provide a statistical description of the acceleration data. The same parameters will also be used to analyze and evaluate the replication quality of the laboratory test simulator. 


\section{Results: Road Measurement}

A nearby road with specific characteristics was selected as the test ground. The pavement on the selected road did not reveal any major cracks or potholes. The surface was nevertheless coarse and provided the desired typical wideband excitation similar to certain roads in Europe.

The average Power Spectral Density (PSD) of the $\mathrm{Z}$ axis acceleration measured for the three speeds is shown in Figure 2.

The frequency spectrums have the same smooth shape showing that there is no strong dynamic behavior of the measurement system or in the excitation input. As expected, the PSD spectrum amplitudes and the rms values increase along with speed despite the fact that the curves cross at around $80 \mathrm{~Hz}$. The maximum difference between the 20 and $36 \mathrm{~km} / \mathrm{h}$ frequency spectrum curves is approximately $4 \mathrm{~dB}$.

Figure 3 shows the waveform amplitude histogram of the measured rear dropout vertical acceleration bandpass filtered between 8-100 Hz with a digital fifth-order zero-phase Butterworth filter. The statistical time parameter amplitudes are also provided in Figure 3. Vertical axis is not shown in these figures because only the shape of the distribution is relevant for this analysis. As the speed increases, a small but noticeable increase in the spread of the base of the distribution occurs. The reduction of the skewness values from 0.14 to 0.08 shows that the excitation becomes more symmetrical at high speeds. The kurtosis does not change significantly with increased speed.

\section{Section 2: Road Simulator Testing Rig}

In Section 1, a technique to acquire an acceleration signal that provides information on road excitation is described. In this section, the signal we obtained is used as an input for a laboratory excitation technique involving the use of a road simulator that was designed and built specifically for bike testing. In this section, we describe the procedure by which we obtained equivalent vertical displacement under the wheels to mimic the road profile, and examine the road replication quality using time and frequency analyses.

\section{Methods}

A dedicated road simulator that was designed and built specifically for bike testing in a previous study [11] is shown in Fig. 4. The lateral equilibrium of the bike and cyclist is provided by attaching horizontal bungees between each 
side of the saddle and a fixed structure. A near-vertical displacement movement is generated under the wheel by the rotation of a long arm that is free to rotate around a pivot provided by two strong bearings. The tire rests on a flat surface. For now, no provision is made to allow the cyclist to pedal during a test. Two D-BOX Kinetron motion actuators produce the required displacement of the swing arm. These actuators were selected because of their flat frequency response, their relative low cost and because they can support a significant DC load. They were originally designed for motion platforms in movie theatre setups. Their effective bandwidth ranges from DC to $100 \mathrm{~Hz}$ and they can provide a peak-to-peak displacement of $2.5 \mathrm{~cm}$ with a maximum linear velocity of $10 \mathrm{~cm} / \mathrm{s}$.

The actuator driving signal is a 16-bit wave file provided by a PC to a USB D-BOX KAI controller. Using the wave file prevents the use of an analog closed loop field replication system that would have adjusted and controlled the analog actuator driving signals in order to obtain the measured in-situ dropout acceleration level. However, the same objective can be achieved in the digital world as described here. First, the Frequency Response Function (FRF) $Q(\omega)=G_{D A}(\omega) / G_{D D}(\omega)$ is measured as the ratio of the cross spectrum $G_{D A}(\omega)$ between the vertical acceleration $A(\omega)$ at the rear dropout of the test bicycle mounted on the simulator, the actuator digital driving signal input $D(\omega)$ (a 16-bit wave file) and the auto spectrum $G_{D D}(\omega)$ of this signal. The same cyclist and same bike used for the road measurements are used for this measurement. The cyclist's posture is also controlled the same way it was controlled for the in-situ measurement. A broadband signal $D(\omega)$ is used for this system characterization phase.

The FRF $H(\omega)=1 / Q(\omega)$ can be calculated. The inverse Fourier transform of $H(\omega)$ corresponds to the impulse response $h(t)$ of the system. To calculate the required driving digital signal actuator input $d(t)$ that would allow the replication of a road signal, the measured road time signal $a(t)$ is convoluted with the impulse response $h(t)$ according to $\mathrm{Eq} 4$.

$$
d(t)=\int_{-\infty}^{t} a(\tau) h(t-\tau) d \tau
$$

The signal $d(t)$ calculated this way generates the required simulated vertical displacement under the bike wheel to mimic the selected road excitation. This signal will recreate the same road displacement excitation regardless of the 
bicycles and cyclists tested. The same procedure could be used for different roads in order to create a road data bank representing different road conditions.

A simple and straightforward way to explore how well this approach reproduces road vibrations simply requires comparing the measured in-situ road acceleration with the acceleration obtained using the simulator driven by $d(t)$. This approach will be used in the next section. Because road excitations are usually not normally distributed [15], frequency domain and time domain parameter estimation $\left(a_{r m s}, s, \kappa\right)$ will be used for analyzing the signal.

\section{Results and Analysis}

Again, the same cyclist and bike used for road measurements were used with the road simulator. The Frequency Response Function $Q(\omega)$ was measured six times using different signal amplitudes covering the full amplitude range of the actuators.

The maximum and minimum FRF curves from all 6 tests are shown in Figure 5 in order to illustrate the system dynamic behavior. The maximum amplitude variation span of the transfer function is less than $1 \mathrm{~dB}$, indicating that the system is stable and behaves linearly.

To evaluate the road simulator replication capability, road measurements at $26 \mathrm{~km} / \mathrm{h}$ were used to calculate the input actuator signals $d(t)$. Due to actuator velocity limits, it was not possible to reproduce a higher speed excitation.

The average PSD of the dropout acceleration for the road signal and the acceleration measured on the simulator at $26 \mathrm{~km} / \mathrm{h}$ are shown in Figure 6. The top graph shows the vertical acceleration and the bottom displays horizontal acceleration. The rms acceleration value is also provided for each test. The road and the simulator vertical acceleration PSD and the rms values are very similar to the curves showing an average of $1 \mathrm{~dB}$ difference between 8-75 Hz. The discrepancy increases with frequency between 75-100 Hz to a maximum $5 \mathrm{~dB}$. For the horizontal axis, acceleration generated by the simulator is lower than the road values with an average difference of $7 \mathrm{~dB}$ and a maximum discrepancy of $13 \mathrm{~dB}$. This is not surprising because there is no horizontal excitation under the wheel. However, the road measurement shows that the horizontal acceleration $\left(a_{\mathrm{rms}}=6.02 \mathrm{~m} / \mathrm{s}^{2}\right)$ is on average half that of the vertical acceleration $\left(a_{\mathrm{rms}}=12.1 \mathrm{~m} / \mathrm{s}^{2}\right)$. 
To obtain a deeper understanding of the replication quality of the simulator, time parameters are calculated. A special plot format [19] is used in Figure 7 to show time characteristics of the signals. Figures 7 a) and c) show the time variation of the road and the lab acceleration for a time duration of $10 \mathrm{~s}$. Plots b) and d) show the respective amplitude histogram. It is worth remembering that these signals are low pass filtered with a cutoff frequency set at $100 \mathrm{~Hz}$ which corresponds to the upper frequency limit of interest. The rms value between the road and the simulated results are very similar. The time signals show however that the simulator does not have the capacity to generate the high peak amplitudes of the road signal. This is clearly demonstrated on the amplitude histogram which shows no count above $36 \mathrm{~m} / \mathrm{s}^{2}$ or below $-39 \mathrm{~m} / \mathrm{s}^{2}$. The kurtosis value of the signal provided by the simulator is lower than the road. This means the acceleration amplitudes are concentrated around the mean. It is believed that the speed limit of the actuator $(10 \mathrm{~cm} / \mathrm{s})$ is in part responsible for this limitation. The skewness value of the road signal is positive and the simulator

is close to zero. This shows that the road provides more upward acceleration than the simulator, which produces acceleration amplitudes more concentrated around the mean.

\section{Discussion}

A bike mounted by a cyclist and towed by a car is the focus of the technique presented in this paper. A different custom-built mechanical system on wheels could have been developed, but the bike is an inexpensive and already existing system that offers several advantages and provides good results.

Having a cyclist mounted on the bike is important because the cyclist's weight prevents the rear wheel from lifting and losing contact with the road. The cyclist also adds damping that calms the dynamic behavior of the system. The accelerometer is installed at the rear wheel dropout in order to be as close as possible to the source of the excitation (i.e. the road) that must be characterized. Preliminary results show that maintaining a constant speed is important in obtaining valid data. When riding a bike, the speed is constantly changing with changes in elevation along the road. The use of the brake is not allowed when taking measurements because it introduces noise in the signal and also because it changes the rear wheel structural dynamic behavior. All of these and previous elements justify having the bike towed by a motor vehicle as a solution.

Despite the inherent non-linear behavior of tires, it is worth noting that for the road signals used in this study, the FRF between the actuator's digital driving signal input and the dropout acceleration shows good linearity. Because of the dominant vertical acceleration amplitude of the bike at the hand and buttock contact points, the main objective of the 
simulator was to duplicate vertical excitation under the wheel. Vertical excitation is frequently used in the automobile industry for studies of noise and vibration harshness. The replication was shown to be excellent up to $75 \mathrm{~Hz}$. The reduction of the replication quality above this frequency as shown in Figure 7 as well as in the reduction of the kurtosis value is related to the speed limit of the actuator. For the current application regarding vibration transmission to the cyclist from a granular rough road, this limitation is not an important factor because $88 \%$ of the road excitation power falls within a 10-50 Hz frequency bandwidth. When studying rough roads with transient events such as cracks, more powerful actuators with a higher upper frequency limit would be required. The skewness value of 0.14 for the measured road vertical acceleration reveals that the bike upward response dominates. This is related to the fact that the road force input to the bike tire can only be upward and only the DC gravitation force can pull the bike down.

\section{Conclusion}

This paper presents a technique to measure a signal input related to the vibration excitation of a road surface. The signal is used to drive a laboratory road simulator that duplicates vertical displacement excitation under a bicycle wheel. For granular roads that provide a broadband excitation within the frequency band of interest, this laboratory technique was shown to provide reliable excitation that adequately duplicates road excitation. The driven signal obtained by this technique will allow the study of dynamic behaviors of different bikes and bike components using the same realistic road profile.

\section{Acknowledgements}

The authors gratefully acknowledge financial support from the National Science and Engineering Council of Canada (NSERC) and the participation of Cervélo and Vroomen-White Design.

\section{References}

[1] Dettori, N and Norvell, D, “Non-Traumatic Bicycle Injuries: A Review of Literature”, Sports Medicine, vol. 36, no. 1, pages 7-12, 2006.

[2] Champoux Y, Richard S. and Drouet J.-M., “Bicycle Structural Dynamics”, Sound and Vibration, July, pages 1622, 2007.

[3] Hastings, A.Z., Blair K.B., Culligan F.K., Pober D.M., "Measuring the Effect of Transmitted Road Vibration on Cycling Performance”, The Engineering of Sport 5, 2004. 
[4] Petrone, N., Giubilato, F., "Comparative Analysis of Wheels Vibration Transmissibility after Full Bicycle Laboratory Tests”, Proceedings of AIAS 40 - Associazione Italiana per l’Analisi delle Solleciaziono, 2011.

[5] IS0 2631-1 (1997), "Mechanical Vibration and Shock -Evaluation of Human Exposure to Whole-Body Vibration -Part 1: General requirements”

[6] Panzke, K.-J and Balasus, W, “Time Dependence and Non-Linearity of the Impedance of the Human Hand-Arm System while Exposed to Intense Vibration”, Int Arch Occup Environ Health, 57 :35-45, 1985

[7] Ewins, D.J., “Modal Testing Theory, Practice and Application”0, 2nd edition, Reseach Studies Press, 2000

[8] Richard, S, “Étude du comportement dynamique d'un vélo de route en lien avec le confort du cycliste”, MSc. Thesis, Université de Sherbrooke, 2005 (French)

[9] Nordstrom, K.-I., “Discomfort Transmission, A Method for Measuring the Comfort of Full-Suspension Mountain Bicycles”, master degree thesis, University of Victoria, 1995.

[10] Richard, S. and Champoux, Y., "Evaluation of Road Bike Comfort Using Classical and Operational Modal Analysis”, Proceedings of IMAC XXIII, 2005.

[11] Brassard, F., “Développement d’un simulateur de vibration pour vélo de route”, master degree thesis, Université de Sherbrooke, under revision, 2010 (french)

[12] Kang, D.-K., Lee, S.-H., and Goo, S.-H., Development of Standardization and Management System fo Severity of Unpaved Test Courses, Sensors, 7, pages 2004-2027, 2007

[13] Sayers, M.-W., and Karamihas, S.-M., "Interpretation of Road Roughness Profile Data”, Federal Highway Administration, Contract DTFH 61-C00143, June, 1996.

[14] Adams, D.E, Janas, J., Goyal, S., Braun, C., Seeniraj, G. and Bono, R.W., “Lab Lessons Learned-Part 2: Road Quality Determination Using Vehicle Data”, Experimental techniques, vol. 30, no. 1, pages: 61-66, 2007.

[15]Bruscella, B., Rouillard, V. and Sek, M.. “Analysis of Road Surface Profiles”, Journal of transportation engineering, January/February, pages 55-59, 1999. 
[16] Imine, H., Delanne, H. and M’Sirdi, N.K., “Road Profile Input Estimation in Vehicle Dynamics Simulation”, Vehicule System Dynamics, 44:4, pages 285-303, 2006

[17] Birdsong, C., “An Integrated Measurement to Road Vibration Simulation System”, Dactron Inc,, 2001.

[18] Miwa, T., "Evaluation methods for vibration effect: Part 3, Measurements of Threshold and Equal Sensation Contours on Hand for Vertical and Horizontal Sinusoidal Vibrations”, Industrial Health, 5, 213-220, 1967

[19] Brandtl, A., “Noise and Vibration Analysis: Signal Analysis and Experimental Procedures”, Wiley, 2011. 
Figures

a)

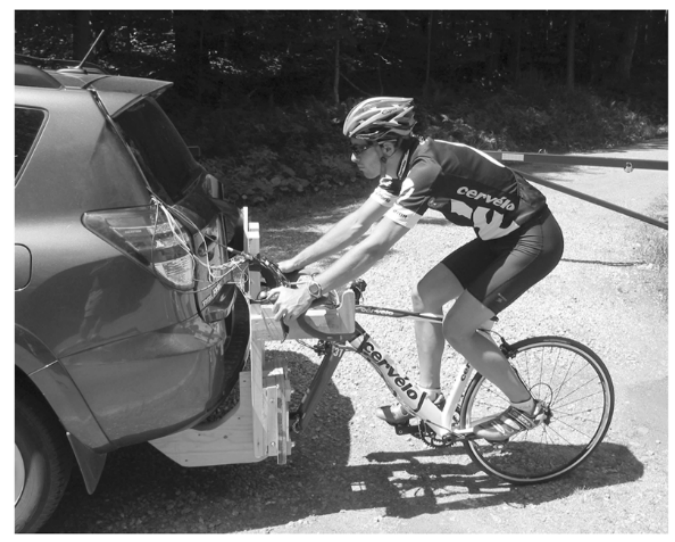

b)

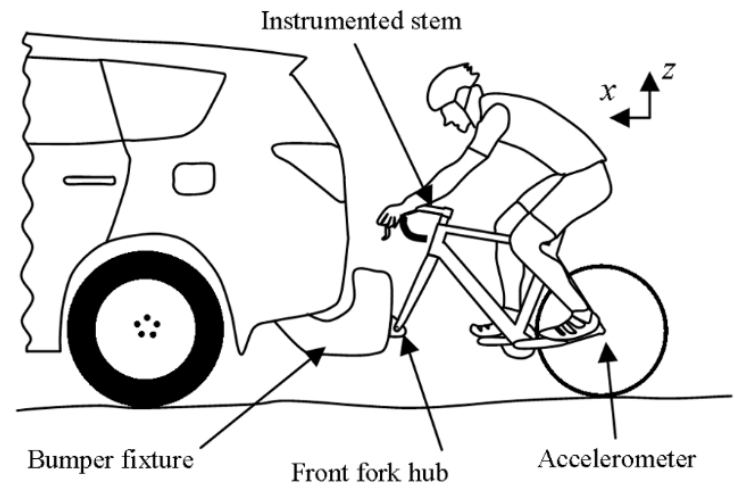

Figure 1: Road data measurement system, a) photographic view b) schematic view

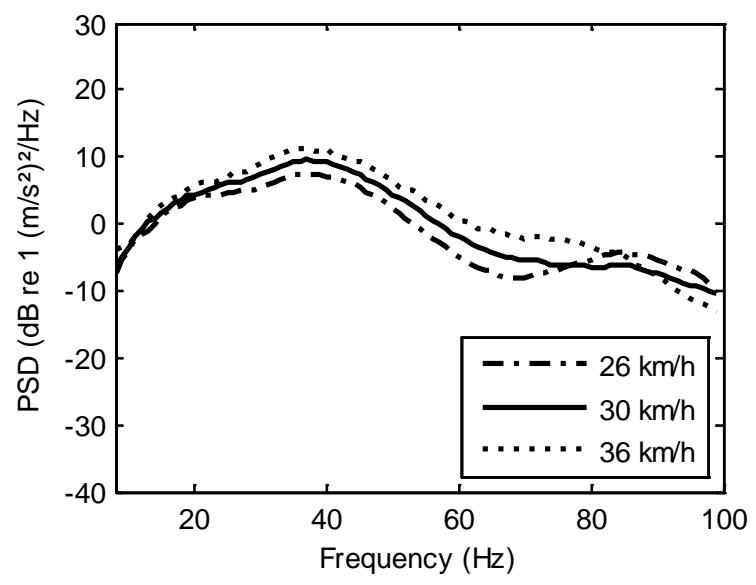

Figure 2: Average PSD for the vertical accelerations at three speeds on the road. Acceleration rms value: 26 km/h: $13.0\left(\mathrm{~m} / \mathrm{s}^{2}\right) ; 30 \mathrm{~km} / \mathrm{h}: 14.6\left(\mathrm{~m} / \mathrm{s}^{2}\right) ; 36 \mathrm{~km} / \mathrm{h}: 17.8\left(\mathrm{~m} / \mathrm{s}^{2}\right)$ 


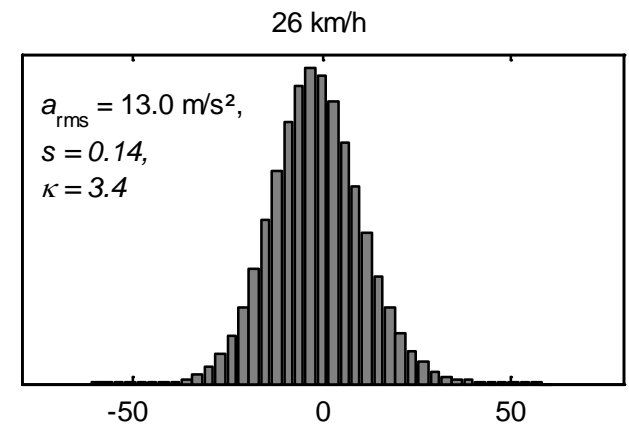

$30 \mathrm{~km} / \mathrm{h}$

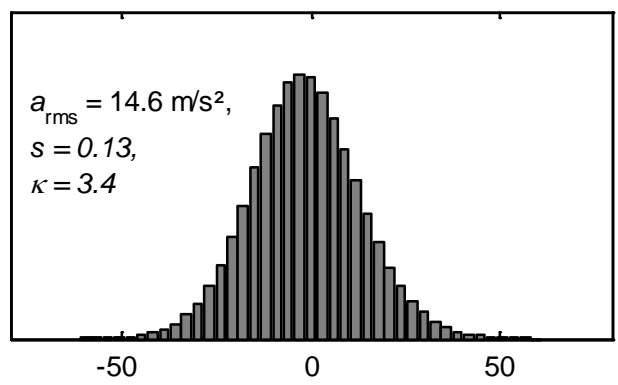

$36 \mathrm{~km} / \mathrm{h}$

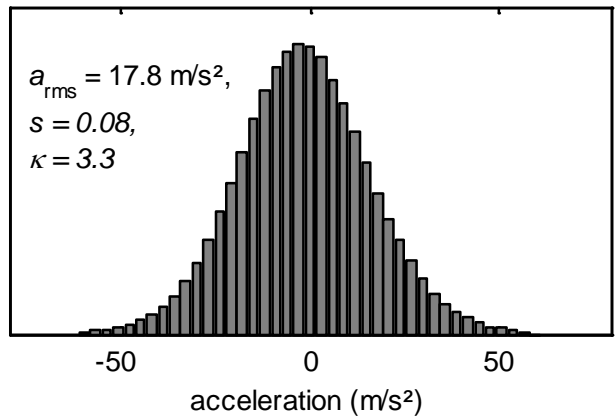

Figure 3: Vertical road acceleration distributions at three different speeds, a fitted normal distribution is superimposed 


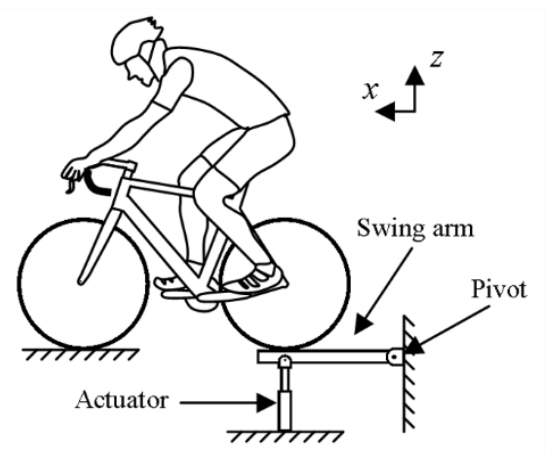

Figure 4: Schematic description of the road simulator exciting the rear wheel

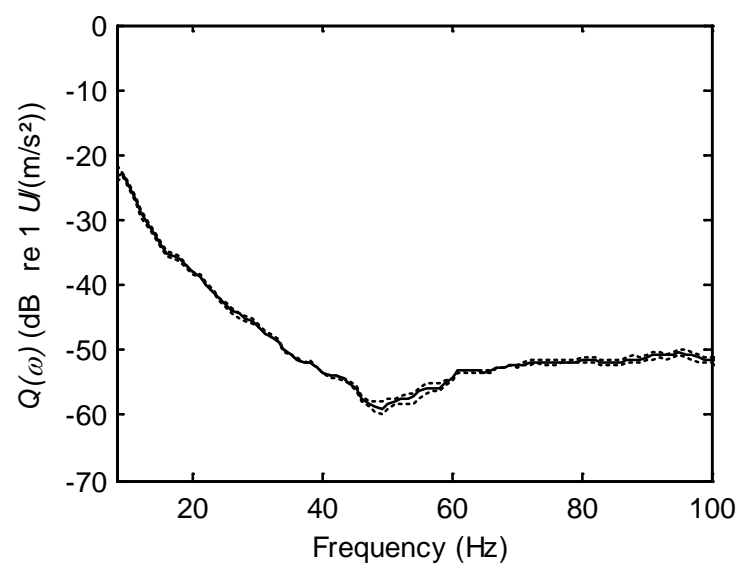

Figure 5: Transfer functions between the simulator input signal and the vertical rear dropout acceleration (Z). Solid line: average transfer function. Dashed lines: minimum and maximum limits of the transfer function. 
a) Vertical acceleration PSD $(Z)$

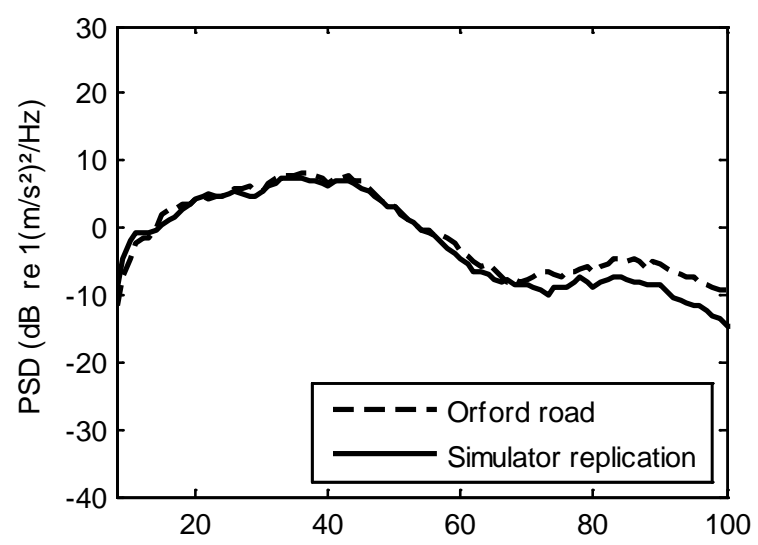

b) Horizontal acceleration PSD $(X)$

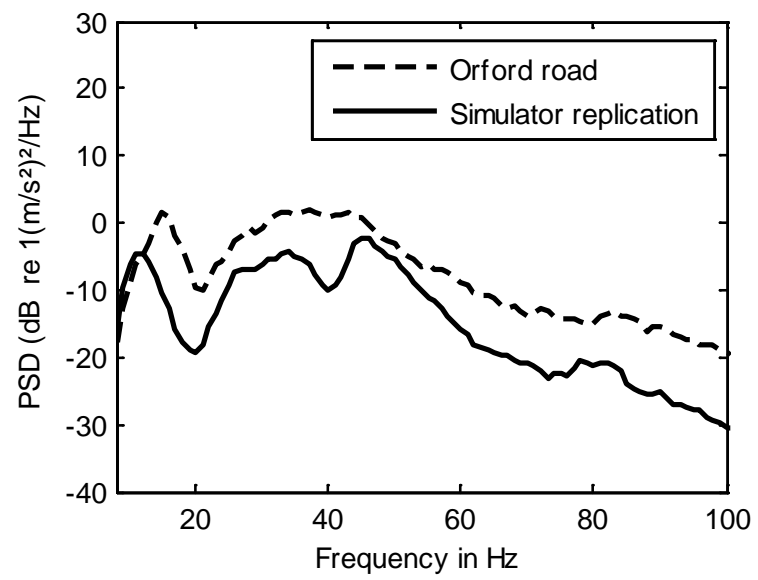

Figure 6: Comparison between the dropout acceleration PSD measured on the road and on the simulator; bike speed $=26 \mathrm{~km} / \mathrm{h}$.

a) Vertical direction $(Z)$; road: $a_{r m s}=13.0 \mathrm{~m} / \mathrm{s}^{2}$; simulator: $a_{r m s}=12.2 \mathrm{~m} / \mathrm{s}^{2}$;

b) Horizontal direction $(X)$; road: $a_{r m s}=6.02 \mathrm{~m} / \mathrm{s}^{2}$; simulator: $a_{r m s}=3.3 \mathrm{~m} / \mathrm{s}^{2}$; 

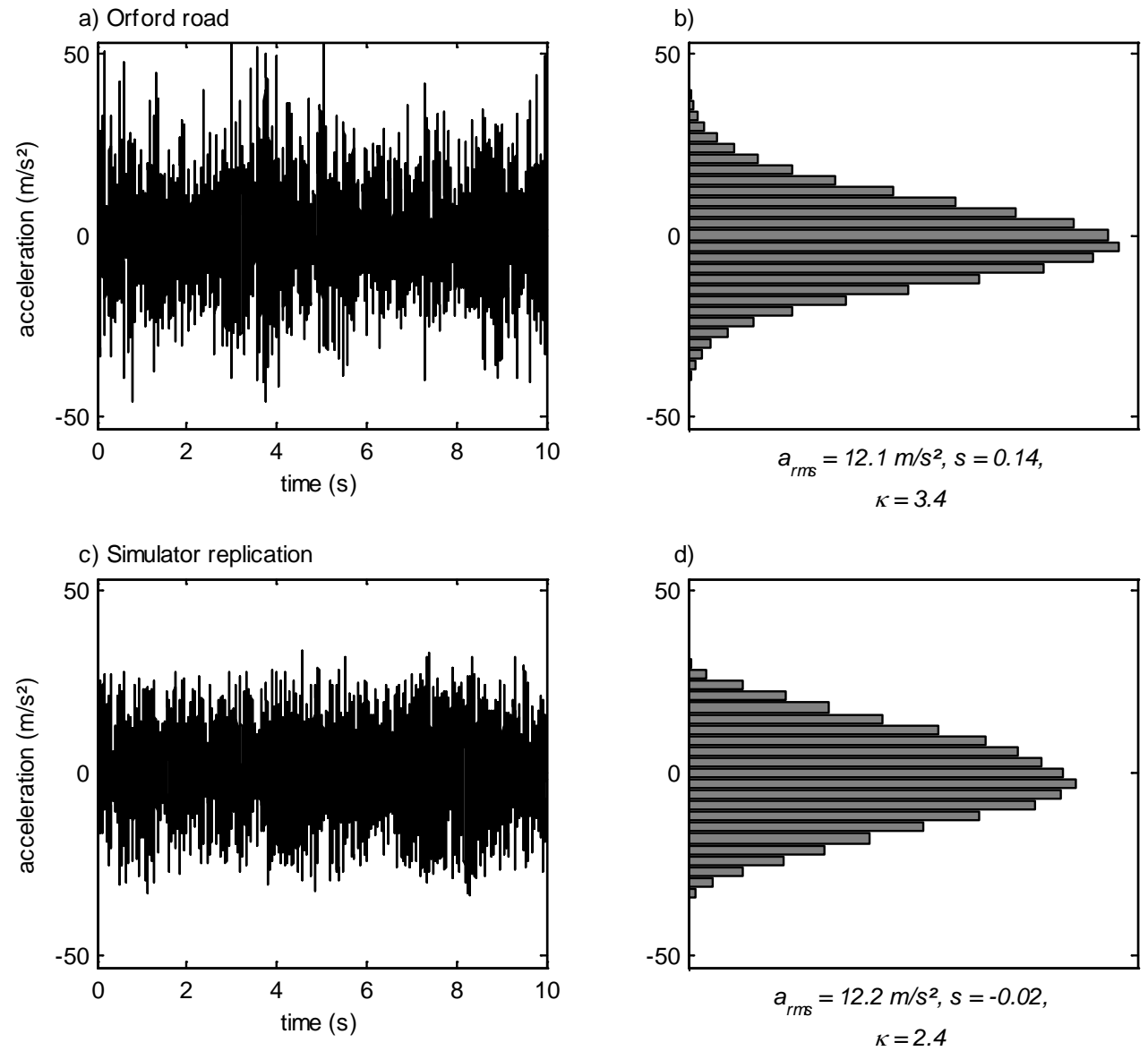

Figure 7: a) Measured road time signal of the vertical acceleration; b) Amplitude histogram of the road signal; c) Simulator replication time signal of the vertical acceleration; b) Amplitude histogram of the simulator replication signal. 